\title{
NOTES AND CORRESPONDENCE
}

\section{Analyzing Altimeter Artifacts: Statistical Properties of Ocean Waveforms}

\author{
G. D. Quartly And M. A. SRokosz \\ Southampton Oceanography Centre, Southampton, United Kingdom \\ A. C. McMillan \\ Southampton Oceanography Centre, Southampton, and Department of Physics, University of Surrey, Guildford, Surrey, United Kingdom
}

9 October 2000 and 18 May 2001

ABSTRACT

\begin{abstract}
In this paper waveforms, that is, returns from the ocean surface, from a number of spaceborne radar altimeter instruments [European Remote-sensing Satellites (ERS-1 and -2), TOPEX, and Poseidon] are examined. This is the first paper to analyze waveform data from a number of altimeters in a consistent manner. Mean shapes and various statistical properties (bin-to-bin correlations, number of independent samples) were determined and the authors comment on their anomalies. The analyses were performed for data over the deep ocean, as that is the best understood surface. However, the determined functional characteristics of the individual altimeters are applicable to their operation over all surfaces. The implications of the existence of these anomalies for the retrieval of geophysical parameters from radar altimeter data are discussed. It is argued that the need for physically based theories, in order to understand radar altimeter returns from the ocean (or indeed any other) surface, implies a need for the engineering and software design of the instrument to be such as to avoid spurious anomalies in the waveforms.
\end{abstract}

\section{Introduction}

Spaceborne radar altimeters have been in use for more than $25 \mathrm{yr}$, and the concepts underlying their operation have been discussed very thoroughly (Chelton et al. 1989). In essence, the instrument emits a short electromagnetic pulse, which is reflected off the earth's surface, and the waveform, that is, the shape and strength of the resultant echo, is recorded on the satellite. The waveform, expressed as a power signal as a function of time, $P(t)$, is the convolution of three terms (Brown 1977; Hayne 1980):

$$
P(t)=P_{\mathrm{PTR}}(t) * P_{\mathrm{FS}}(t) * q(t),
$$

where $P_{\text {PTR }}$, the point target response (PTR), represents the original pulse; $P_{\mathrm{FS}}$ is the flat surface response, which includes the antenna pattern of the altimeter; and $q(t)$ represents the probability distribution function (PDF) of the heights of the reflecting surface, expressed in terms of two-way travel time. The emitted pulse may be approximated by a Gaussian function, and if the PDF of

Corresponding author address: Dr. Graham D. Quartly, Southampton Oceanography Centre, Empress Dock, Southampton SO14 3ZH, United Kingdom.

E-mail: gdq@soc.soton.ac.uk reflecting facets is also Gaussian, the waveform should be as illustrated in Fig. 1a, where a level of thermal noise has also been added.

Short-duration large-amplitude pulses would produce too high a peak power for the amplifiers; in practice, all recent altimeters have used long-duration chirps with the same frequency content as the idealized pulse, but a much lower peak power. Using predictive software (known as an $\alpha-\beta$ tracker) the receiving chain provides a second chirp at the expected time, which is mixed with the return echo. The resultant signal is thus a function of the difference frequency, and its properties depend not only on the particular part of the earth's surface underneath but also the characteristics of the various frequency filters used in the chirp generation and "deramping." Further details on the actual implementation may be found in Chelton et al. (1989), Zieger et al. (1991), and Marth et al. (1993).

Figure 1a shows an ideal waveform; those recorded in practice are somewhat different (Fig. 1b). First, use of discrete Fourier transforms (DFTs) in the processing on board the satellite leads to "wraparound," that is, a reduction in power in the last few bins and a commensurate rise at the beginning of the waveform. The other clear feature is the Rayleigh or "fading" noise, due to the incoherent addition of signals from different reflect- 


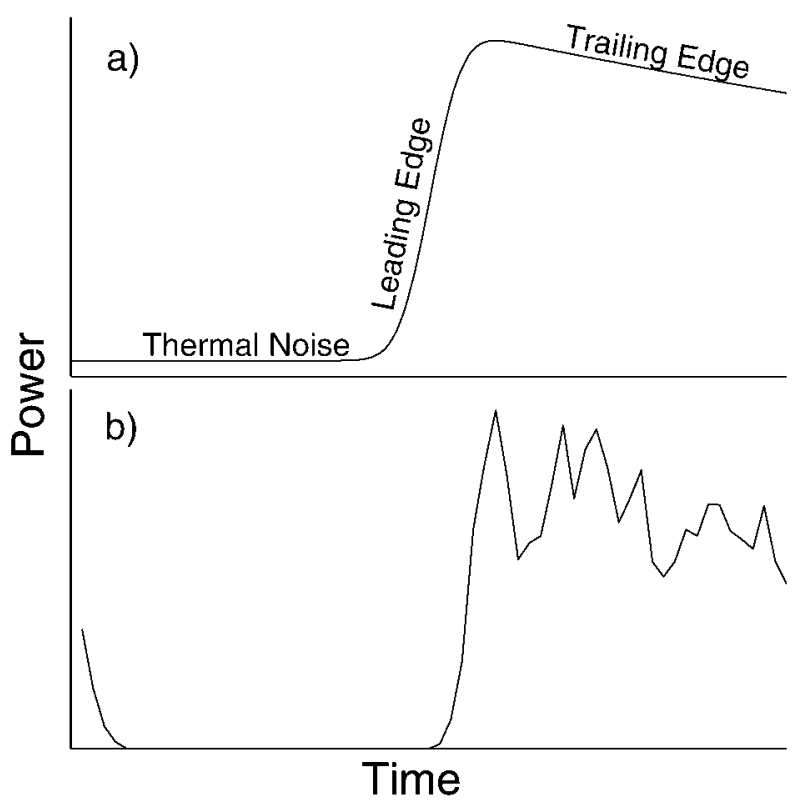

FIG. 1. (a) An ideal waveform from an ocean surface. (b) Actual waveform, showing wraparound and Rayleigh noise (ERS-2 0.05-s average).

ing facets. For each bin of a single pulse, the received power will be governed by a negative exponential PDF. When an altimeter averages $N$ such independent returns together the power will conform to a chi-squared distribution with standard deviation (std dev) equal to the mean divided by $\sqrt{N}$. For an ideal altimeter traversing a surface of constant geophysical properties the expected power in each waveform bin should be similar to that depicted in Fig. 1a, but with fading noise on each realization. If one determines the mean observed value at a bin, and the std dev about this mean, then $\alpha$, the ratio of the mean to the std dev will be related to $N$ by

$$
\alpha=\sqrt{N} .
$$

If the bin separation is greater than the effective pulse width, the power received in successive bins will come from different reflecting facets and should be independent. On that basis, the anomalies (i.e., power relative to the mean for that bin) should be totally uncorrelated with those in any other bin.

The purpose of this paper is to examine waveform data from a number of recent altimeters and evaluate these three properties-shape of mean waveform, effective number of independent pulses, and bin-to-bin correlation of anomalies. Although some of these anomalies are documented [e.g., see the discussion of Hayne et al. (1994) and Rodríguez and Martin (1994a) regarding Ocean Topography Experiment (TOPEX) $\mathrm{K}_{\mathrm{u}}$-band waveforms], others have not been reported previously in the open literature. Here we provide a comparison of the waveform quality from a number of recent instruments. The methodology is detailed in section 3, with the results in the succeeding section. However, first we discuss the various uses of waveform data, and their sensitivity to waveform artifacts. Although our analyses are only of deep ocean data, section 2 summarizes applications over lakes, rivers, rain, ice, and land since our results pertain to the operation of altimeters over all terrains.

\section{Applications of waveform data}

Radar altimeters have been designed with the aim of measuring sea surface height as accurately as possible. In terms of altimeter footprints of $\sim 10 \mathrm{~km}$ across (Chelton et al. 1989), the oceans usually provide a locally homogeneous flat surface. This allows a relatively wide antenna beamwidth to be used (see Table 1), as the instrument is in "pulse-limited" mode; that is, only the earliest returns (those from nadir) are recorded. The simple PDF of reflecting facets and local homogeneity lead to a tractable form for the average waveform (see Fig. 1a).

Radar altimetry has become a standard "tool" for measuring certain geophysical parameters of the ocean surface: in particular, the sea surface height $h$, the significant wave height $H_{\mathrm{s}}$, and the roughness of the sea surface $\sigma^{0}$, from which wind speed is inferred (Brown 1977; Chelton et al. 1989). The Brown model has been the basis of all altimeter algorithms to date. It has been modified to allow for nonlinear waves (and therefore non-Gaussian statistics) by Lipa and Barrick (1981) and Srokosz (1986), which allows other wave parameters, such as skewness, to be derived from the shape of the returns (Tokmakian et al. 1994). The derivation of geophysical parameters from the radar return takes one of three forms: an empirical, a semi-empirical, or a physically based algorithm. Lipa and Barrick (1981) have attempted to determine the skewness by a direct deconvolution of the waveform data, minimizing the effects of fading noise by averaging data in 6- or 24-s segments. The effect of cross-skewness is similar to mispositioning of the waveform within the window (Rodríguez 1988), and joint estimation of skewness leads to greater errors in the height (Rodríguez and Chapman 1989). For such deconvolutions it is necessary to have an accurate estimate of the true PTR rather than using the idealized form (Rodríguez 1988). Ideally, a physically based algorithm should be used, as this provides a correct understanding of the relationship between the geophysical parameter of interest and the radar return from the ocean surface. In practice, the algorithms used for deriving geophysical parameters from radar altimeter data are of all three types mentioned previously (see, e.g., Tokmakian et al. 1994 and Hayne et al. 1994). The desire for increasingly accurate estimates of geophysical parameters has led to a greater emphasis on physically based algorithms. Such algorithms need to take account of both the physics of radar 


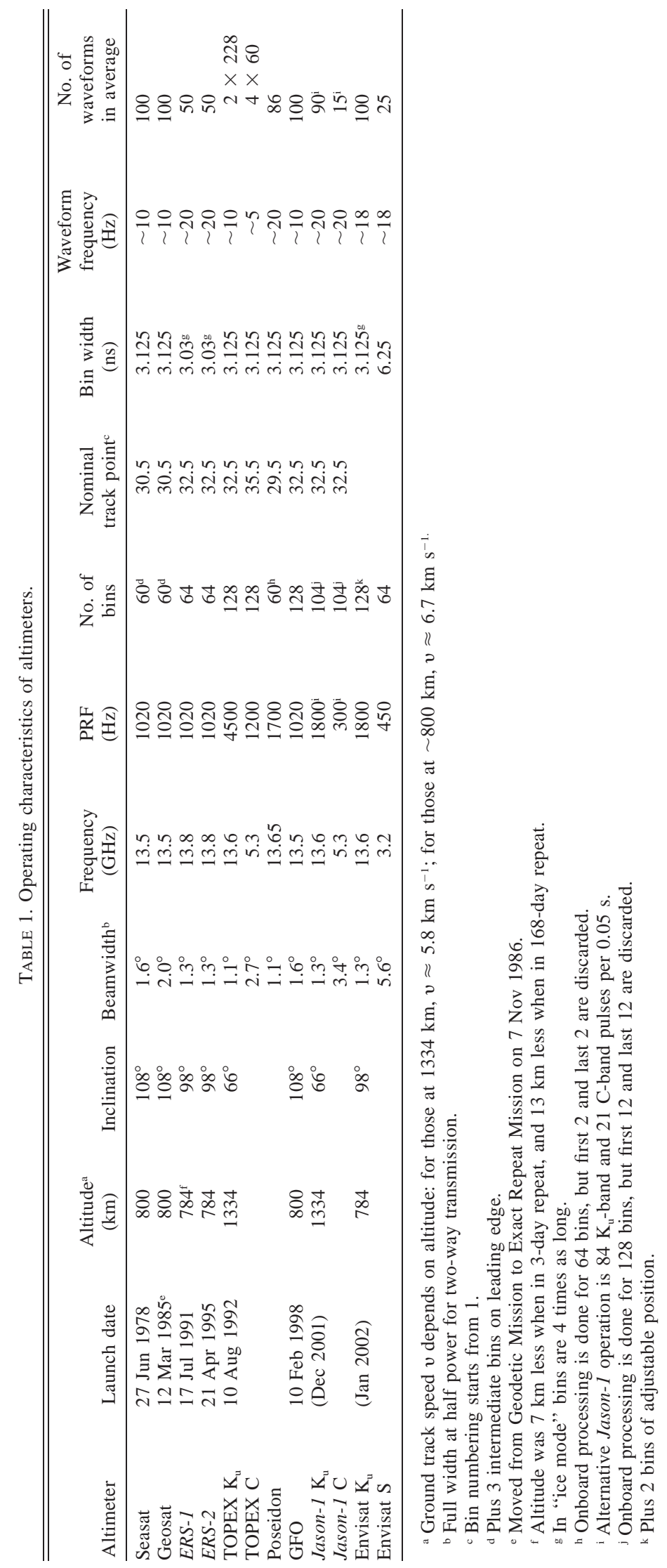


interaction with the ocean surface and the characteristics of the radar altimeter itself.

"Oceanlike" waveforms may also be returned from large rivers and lakes. When this occurs the mean water height may be retrieved in the same manner as for deep ocean applications. Care is required in the processing to pass as many waveforms as possible, but edit out any that are significantly affected by the higher land surfaces. Similar problems arise when considering ocean sections right into the coastal zone (Brooks et al. 1990). There are also the additional problems of narrow-peaked waveforms due to very calm conditions (see, e.g., Birkett 1994) and that the waveforms may not be centered at the track point, and so waveform artifacts far from the nominal tracker point can be of greater concern.

Because the presence of atmospheric liquid water (both clouds and rain) can attenuate microwave radiation, it too may affect altimeter waveforms. The most pronounced effects are for heavy rain concentrated in a small area, as then the attenuation is not uniform throughout the waveform (Walsh et al. 1984). The effect can only be seen over oceans where there is a clear expectation of the shape of the rain-free waveform. Examples may be found in data from the first European Remote-sensing Satellite (ERS-1; Guymer et al. 1995) and TOPEX (Quartly 1997). On account of its two frequencies, TOPEX offers much greater scope for quantitative recovery of the strength and size of rain cells (Tournadre 1998; Quartly 1998). Because such methods are based on the differences between the signals at the two frequencies and the anomalies in the $\mathrm{K}_{\mathrm{u}}$-band waveforms relative to their expected rain-free shape, it is important to understand all the artifacts within the waveform. As rain events are small there is no recourse to along-track averaging of waveforms to reduce the effect of fading noise. Thus, it is hoped that these effects are minimized through optimal design and construction.

Recently an advance has been made in the separation of waveforms reflected off sea-ice from those off water in the leads in between (S. W. Laxon and N. Peacock 1999, personal communication). This has not only led to studies of tides and mesoscale variability in the icecovered seas through application of standard altimetric techniques, but also enabled satellite monitoring of the thickness of the sea-ice.

Compact snow is another surface that provides characteristic returns. In this case there is scattering from both the interface and the interior of the snow. This leads to different waveform shapes according to the relative contributions of surface and volume scattering, which vary with the compactness and the grain size of the snow (Ridley and Partington 1988). These differently shaped waveforms necessitate the use of different algorithms for tracking the surface returns. Legrésy and Rémy (1998) have shown how temporal variations in the compactness and grain size lead to systematic errors in $\sigma^{0}$ and the leading edge width, which in turn affects the height estimation. To determine ice shelf changes to a high accuracy Wingham et al. (1998) used local regressions of height on $\sigma^{0}$ that in part compensate for this effect. Furthermore, Legrésy et al. (1999) have shown that the orientation of the altimeter polarization with respect to the wind-generated sastrugi (snow dunes) can also significantly affect the degree of volume scattering and thus lead to different waveform shapes and derived parameters according to the direction of the altimeter's transect. Because ice surfaces cannot be assumed to be flat one has to allow for altimeters ranging as far as $1^{\circ}$ from nadir and apply the appropriate slope corrections (Brenner et al. 1983). There may also be reflections from two distinct surfaces. Algorithms have been developed to overcome some of these problems (Bamber 1994), but their performance will be degraded if there is variable gain along the waveform or correlation between anomalies in different bins.

Altimetry over land is even more complicated. Although the tracker on ERS-1 maintains lock over land on $\sim 80 \%$ of occasions (Berry 1999), it is not always possible to derive meaningful values from those data. The altimeter usually tracks well over marshes, deserts, and rain forests (Guzkowska et al. 1990), and various authors (e.g., Cudlip et al. 1994; Guzkowska et al. 1990) have investigated the use of such homogeneous regions as reference surfaces for monitoring altimeter performance. Using a variety of different tracker algorithms, Berry (1999) has determined land elevations, achieving a good repeatability between different passes. This has in turn revealed the inconsistencies in various digital elevation models obtained from conventional surveying techniques.

Anomalies in altimeter waveform data can degrade their usefulness in all these applications. However, in this paper we study the statistical properties of waveforms over the oceans, as their homogeneity provides a simple expected form, allowing us to compare different altimeters.

\section{Calculation of waveform statistics}

In order to assess the magnitude of the sampling noise (and thus the number of independent pulses averaged) and the correlations between different bins, it is necessary to calculate anomalies relative to a mean waveform shape. Although the onboard automatic gain control (AGC) applies a variable attenuation to the return signal so that it will fit the dynamic range available in the detector, the correlation analysis cannot be done using a global mean waveform, as the mean shape will still vary with wave height, wind speed, etc. One solution is to fit an expected oceanlike return to each waveform and calculate anomalies relative to that; however, that process is affected by how well the received waveforms compare to the ideal expected, which is the objective of this paper.

Instead we use the method of ensembles, previously advocated by Rodríguez and Martin (1994b), in which 


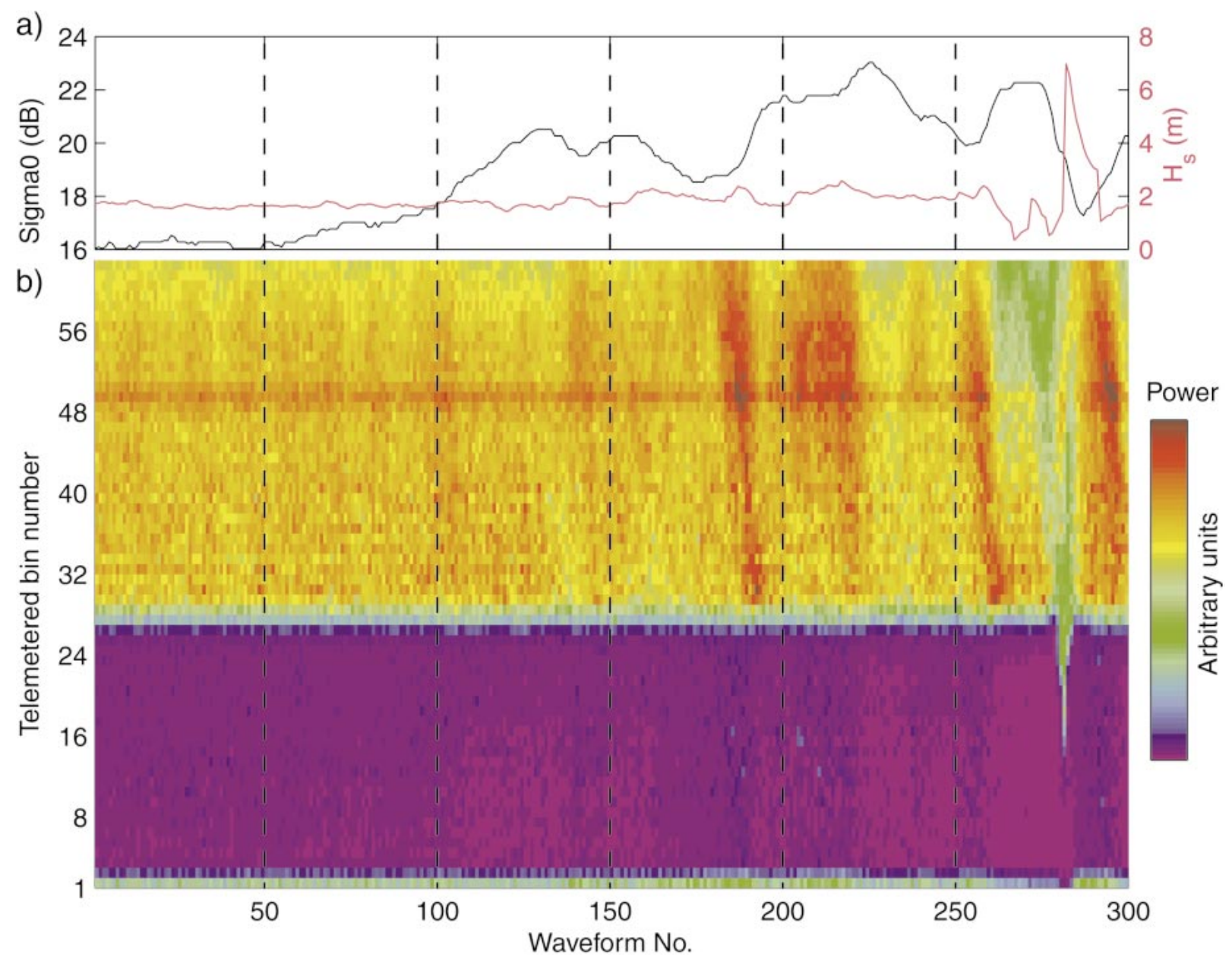

FIG. 2. Variations in data, illustrated with TOPEX C band, prior to editing for ensemble statistics. (a) Backscatter strength and wave height. (b) Power in each individual waveform bin.

the data stream is broken up into short segments, a local mean determined for each ensemble, and then the statistics for the ensembles averaged together. Clearly there is a trade-off between the robustness of the statistics for an ensemble (dependent upon how many average waveforms it contains) and the assumption that the underlying conditions have not changed. Here we choose 10 -s ensembles for each altimeter, albeit that number of average waveforms per second varies by a factor of 4 between instruments (see Table 1). The onboard tracker attempts to position the receive window such that the half-point of the leading edge lies at the desired tracker point (see Table 1). The tracker is usually successful at this, but given that the positioning is based on predictions, there is some variation in the waveform position, which will clearly enhance the relative magnitude of the variations on the leading edge. This paper is not seeking to address the positioning of the waveforms and thus the performance of the different trackers, but rather the shape and correlations within the waveform data.

An example from TOPEX-C is shown in Fig. 2 to illustrate the data editing that also has to be applied. All the waveforms within the first two ensembles appear to be from the same distribution and thus the means for each of those ensembles provide suitable references for the calculation of anomalies. There is significant variation in $\sigma^{0}$ during the third and fourth ensembles, with strong positive anomalies providing a coherent variation in the fourth and fifth ensembles. In the middle of the sixth ensemble there is a strong power deficit that migrates to the leading edge of the waveforms and then causes the tracker to misposition the waveforms, leading to an excess of power prior to the nominal track point (bin 27.5). Such power deficits have been associated with an altimeter passing close to a strong rain cell (Quartly 1997, 1998; Tournadre 1998).

Therefore, the $N_{1}$ pulses within an ensemble were tested to see whether their half-power point was within three bins of expected location, and that the $\sigma^{0}$ was within $0.1 \mathrm{~dB}$ of the median for the ensemble, and that $H_{\mathrm{s}}$ values were within $0.5 \mathrm{~m}$ of the ensemble's median. These acceptance criteria are fairly broad for the $\sim 60$ - 
$\mathrm{km}$ data segments used, but will occasionally discard extreme values that are part of the normal distribution. However, their function is to remove strange geophysical signals such as sea-ice, rain, or rapidly changing wind/wave fields. If the number of waveforms passing these editing criteria, $N_{2}$, exceeds $40 \%$ of $N_{1}$, then these waveforms were used to calculate a mean waveform and anomalies relative to that mean. [We also investigated the use of waveform peakiness (Laxon and Rapley 1987) as an extra editing criterion, but found it made little change to the tests discussed above.]

For each valid ensemble we calculated and stored a mean $m$, standard deviation $s$, and correlation $c$ calculated as follows:

$$
\begin{aligned}
m(i) & =\left(1 / N_{2}\right) \sum_{k=1}^{N_{2}} w_{k}(i) \\
s(i) & =\left\{\left(1 / N_{2}\right) \sum_{k=1}^{N_{2}}\left[w_{k}(i)-m(i)\right]^{2}\right\}^{0.5} \\
b(i, j) & =\left(1 / N_{2}\right) \sum_{k=1}^{N_{2}}\left[w_{k}(i)-m(i)\right]\left[w_{k}(j)-m(j)\right] \\
c(i, j) & =b(i, j)[b(i, i) b(j, j)]^{-0.5},
\end{aligned}
$$

where $i, j$ are bin numbers and $k$ indicates waveform number.

In all cases we used the values of $H_{\mathrm{s}}$ and $\sigma^{0}$ (or AGC) supplied with the data, rather than making our own independent estimates. The mean value of $H_{\mathrm{s}}$ for each ensemble was also stored. Typically the amount of data used from each altimeter corresponded to one day's collection. The statistics for all ensembles within $10 \%$ of a nominal $H_{\mathrm{s}}$ value (e.g., $3 \mathrm{~m}$ ) were then combined, with $N_{3}$, the number of such ensembles, being of order 10 for $H_{\mathrm{s}}=1 \mathrm{~m}$ or $H_{\mathrm{s}}=5 \mathrm{~m}$, but considerably more for intermediate values. Because the ratio of mean to standard deviation could be sensitive to very occasional low values of $s$, the mean ratio of the ensembles $\alpha$ was determined via reciprocals, as this was found to be slightly more robust:

$$
1 / \alpha(i)=\left(1 / N_{3}\right) \sum_{k=1}^{N_{3}}\left[s_{k}(i) / m_{k}(i)\right] .
$$

(Examination of particular collections of ensembles showed this definition to give a robust average, without significant bias.) Note that although antenna mispointing is known to affect the waveform shape (especially in the later bins), this will not affect the results of the processing detailed here. This is because mispointing is a phenomenon that changes on a very large scale (of the order of an orbit), and thus will have negligible variation within any 10 -s ensemble.

\section{Results}

Here we present the results for each individual altimetric dataset, commenting first on the shape of the mean waveforms; then on $\alpha$, the ratio of the mean waveform to its standard deviation; and finally on the degree of correlation between the anomalies at successive bins.

\section{a. ERS-1}

ERS-1 was launched on 17 July 1991 into a high inclination orbit to allow nearly global coverage. It carries a varied package of sensors, intended for multidisciplinary research. Among these is the $\mathrm{K}_{\mathrm{u}}$-band radar altimeter, which emits 1020 pulses per second and averages them in groups of 50 (Francis et al. 1991). In standard ocean-tracking mode the waveform is recorded in 64 bins of width $3.03 \mathrm{~ns}$, with the tracker aiming to position the half-power point at bin 32.5 (i.e., midway between bins 32 and 33). (There is also an ice-tracking mode in which the 64 bins are four times as wide, which gives the tracker greater agility in crossing the steep gradients of Antarctica and Greenland.) For the ERS altimeters, the received signal is multiplied by a "Hamming window" (see p. 4-2-27 of ESA Document 1993). This is equivalent to convolving the voltage of the Fourier transformed signal by a three-point smoother [0.42, $1,0.42]$, the effect of which will become clear later.

Figure 3a shows the mean waveform for ERS-1 for three different values for $H_{\mathrm{s}}$. All show the generally expected shape, with broadening of the leading edge as $H_{\mathrm{s}}$ increases, and a tail-off along the trailing edge that is $H_{\mathrm{s}}$-independent. In these waveforms, which have had no external bin-dependent corrections applied, a slight "waviness" can be discerned along the trailing edge. A similar waviness can be discerned in the characteristics of the intermediate frequency (IF) filter (see p. 9 of Francis 1990). The characterization of this filter in that document shows roughly the same wavelength as observed in Fig. 3a (8 bins), but is larger in magnitude. Given that the filter characteristics may have changed slightly through space exposure/aging, and that slight delay variations will smear and thus reduce the ripple amplitude, the waviness observed in Fig. 3a may be considered consistent with the frequency response of the IF filter. There is a pronounced wraparound, which affects the first four and last four bins. This is due to the onboard DFT, and the lack of sharpness of the cutoff of the IF filter; this effect is particularly pronounced for the ERS altimeters on account of the three-point smoothing. There appears to be no thermal noise; this is due to a simplification in the onboard processing. This occurs due to the way the onboard processor handles the averaging of the 50 waveforms (details may be found in ESA 1993, p. 4.2-27, footnote 6). In order to prevent overflow of the registers in which the values for the power in the bins are stored, rather than add up 50 values in each bin and then divide by 50 to obtain the average, each value is divided by 50 and then added. Arithmetically these operations are identical, but numerically the division by 50 of the values in the bins before the leading edge leads to a loss of precision and reduces the 


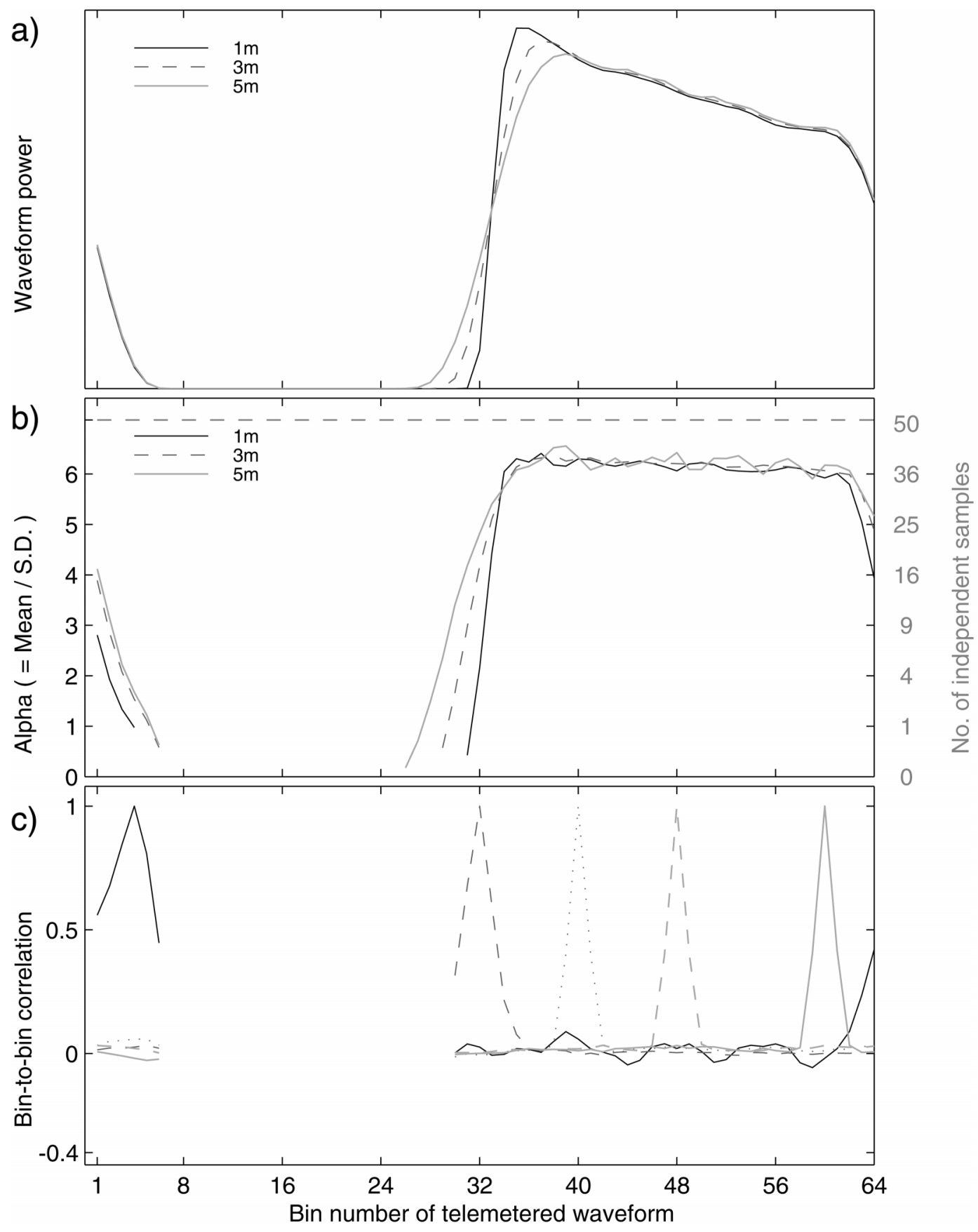

FIG. 3. ERS-1. (a) Shape of mean waveforms for varying $H_{\mathrm{s}}$ values. (b) Ratio of mean to standard deviation of ensemble; right-hand scale shows equivalent number of independent pulses. The horizontal dashed line shows the instrument limit (number of actual pulses averaged). (c) Bin-to-bin correlations (for $H_{\mathrm{s}}=2 \mathrm{~m}$ ). (Each correlation curve is identified by having a correlation of unity with itself.)

apparent power there to zero. Of course there is thermal noise contributing to the perceived power level in the second half of the waveform. The absence of information in the first half precludes an estimate of how large the thermal contribution is and affects the ability of advanced techniques, such as maximum likelihood estimation, that attempt to determine all the waveform parameters at once (Tokmakian et al. 1994).

The standard deviation of anomalies about the local mean (not shown) has essentially the same shape profile as the mean. Figure $3 b$ shows $\alpha$, the ratio of the mean to the standard deviation [see Eq. (4)], with the effective 
number of independent pulses [see Eq. (2)] given by the nonlinear scale on the right. There are no values in the thermal noise region because of the absence of data. The effective number of independent pulses is $\sim 36$ ( $\alpha$ $=\sim 6.0$ ) throughout the trailing edge. The shortfall from the expected value is principally due to the truncation used in the averaging process, the effect of which depends upon the data range employed. We simulated the situation by generating random numbers according to a negative exponential distribution, with known mean and rounding the values down to the nearest integer. When the mean is of the order of eight counts (i.e., corresponding to $\sim 400$ before division by 50 ), then the effect of simply truncating to integral values leads to a value for $\alpha$ of 6.6 instead of 7.1. The reduction in $\alpha$ is greater when the number of counts is smaller.

On the leading edge there is a sharp reduction in values as one moves to earlier bin numbers. This is consistent with the concept that the contributing returns are coming from a smaller disc on the sea surface [as noted for Geosat data by Rodríguez and Martin (1994b)] and also the increased effect of data truncation at low count values. However, movement of the whole waveform within the window, as the tracker attempts to position the middle of the window at the predicted halfpower point, can increase the standard deviation and thus reduce $\alpha$.

Figure $3 \mathrm{c}$ shows the correlation between bins within individual waveform averages. Once more, no values can be provided for the thermal region. The trailing edge, again the simplest region to interpret, shows a strong correlation (about 0.4 ) between the anomalies at one bin and that adjacent to it, but no large-scale correlations. Calculations show that the three-point smoother specified in section 4a should produce a correlation of 0.33 between nearest neighbors. The extra correlation observed may be due to the actual PTR being broader than in ground tests. There is a strong correlation (not shown) between the anomalies at the end of the trailing edge and those at the very beginning of the waveform, due to these being linked by the wraparound effect of the DFT.

\section{b. ERS-2}

ERS-2 was launched on 21 April 1995, bearing an almost identical altimeter to ERS-1. Although ERS-1's problems with rounding in integer arithmetic had been identified prior to $E R S$-2's launch, it was not possible to change the onboard processing in a radical manner. The only change was that the AGC preset value was reduced by $3 \mathrm{~dB}$, effectively doubling all the values in the bins prior to the division by 50 and summing (M. Roca 1998, personal communication). However, this was not sufficient to aid recovery of information from the thermal noise region. The waveform statistics for $E R S-2$ (Fig. 4) are essentially the same as for its sister instrument, except that $\alpha$ reaches a plateau of $\sim 6.5$ for all wave heights (consistent with a reduction in the effect of truncation). It is clear that ad hoc "fixes" do not necessarily solve the problem, though they may provide some improvement.

\section{c. TOPEX/Poseidon}

The TOPEX/Poseidon satellite was launched on 10 August 1992 to perform a dedicated mission measuring the oceanic circulation (Fu et al. 1994). To this end it had a high altitude (to minimize atmospheric drag and improve orbit computation) and an orbital inclination of only $66^{\circ}$ (sufficient to cover nearly all of the ice-free seas). The platform houses two different altimeters. TOPEX is a high performance instrument with a high pulse repetition frequency (PRF) and the novelty of operating at both $K_{u}$ band and $C$ band (in order to enable better corrections for the ionospheric path delay). These pulses were cleverly interleaved so that the antenna was emitting $\mathrm{K}_{\mathrm{u}}$-band pulses while receiving C-band pulses and vice versa (Zieger et al. 1991). Conventional (traveling wave tube) technology is used for the amplification at the $\mathrm{K}_{\mathrm{u}}$ band, but solid-state amplifiers are used at $\mathrm{C}$ band. Here we treat TOPEX- $\mathrm{K}_{\mathrm{u}}$ and TOPEX-C pulses separately.

The other altimeter on the platform is Poseidon, a single frequency ( $\mathrm{K}_{\mathrm{u}}$-band) device with solid-state amplification (Raizonville et al. 1991). As TOPEX and Poseidon share a common antenna they cannot be operated simultaneously; Poseidon (as the experimental instrument) is on for $10 \%$ of the time, with TOPEX on for the remainder. The TOPEX waveform data are from 1993, which is after the problems with mispointing of the platform (Fu et al. 1994), but before the degradation of the A side of the TOPEX altimeter became apparent.

\section{1) TOPEX-K $K_{u}$}

In each 53-ms interval, TOPEX emits a burst of 228 $\mathrm{K}_{\mathrm{u}}$-band pulses, whose echoes are received in 128 bins of width $3.125 \mathrm{~ns}$, with the onboard tracker attempting to position the half-power point at bin 32.5. [The extension to 128 bins was principally for the estimation of antenna mispointing from the waveform data, as no bins beyond 64 are used in the calculation of $\sigma^{0}$ or $H_{\mathrm{s}}$. However, these extended waveforms have also been useful in studies of the size of rain cells (Tournadre 1998; Quartly 1998).] Prior to transmission those waveform data are reduced in two ways. First, successive bins are averaged together-the first 16 in pairs to give eight values, the next 32 are transcribed directly, the subsequent 16 in pairs again, and the last 64 in quartets. This leads to a reduction in the number of bins to 64 , with the effective tracker point now at 24.5. Second, successive mean waveforms in time are averaged together to give 64-bin telemetered waveforms resulting from the averaging of 456 pulses each. Although the AGC attenuation control can only be applied in steps of $1 \mathrm{~dB}$, 


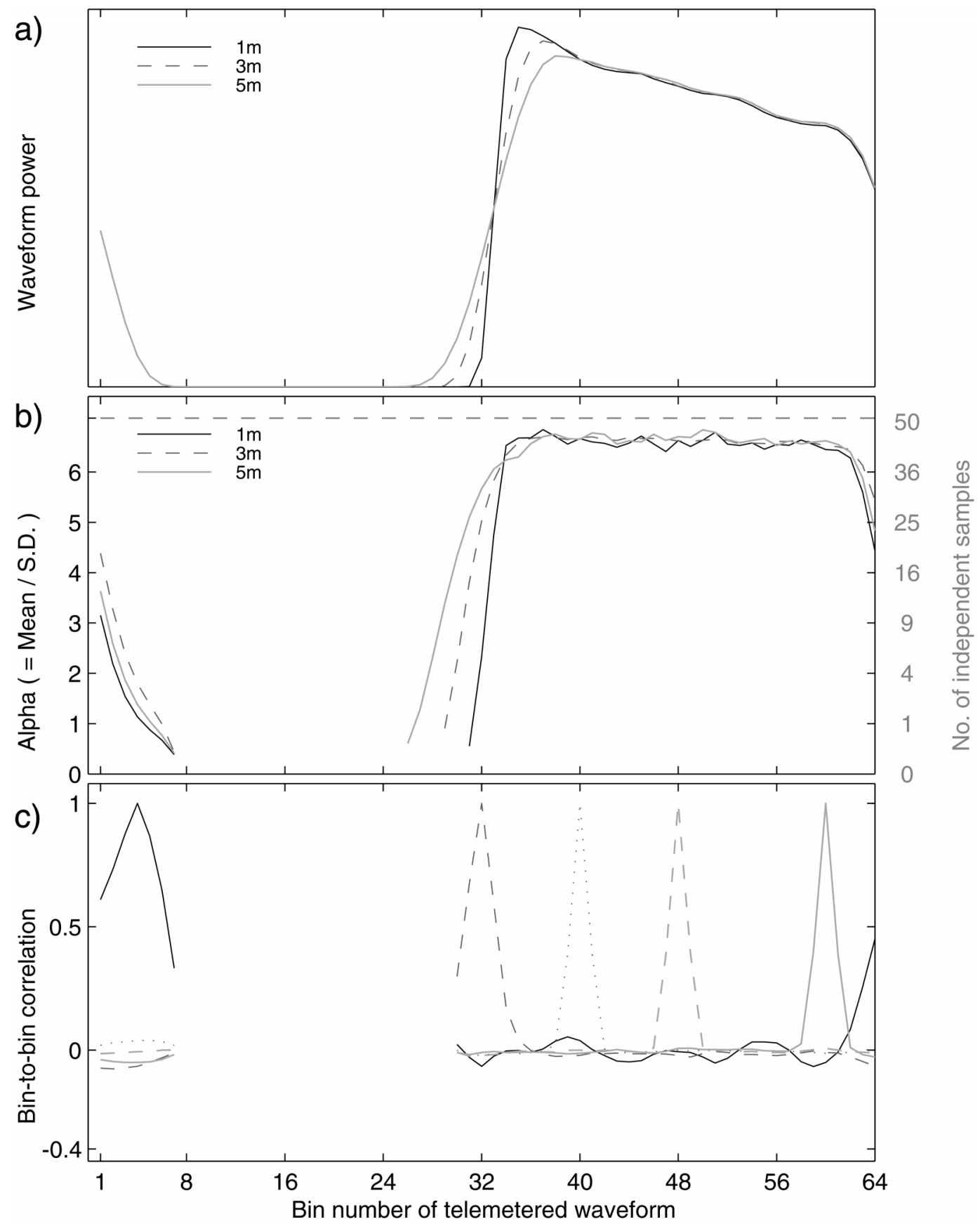

FIG. 4. ERS-2. (Description same as for Fig. 3.)

it can adopt different values for pulses within a burst, giving a much finer resolution for the mean AGC applied. Unfortunately, the mean value applied was rounded to the nearest $0.25 \mathrm{~dB}$ prior to telemetry, leading to a loss in finescale variations, which have hampered applications needing accurate $\sigma^{0}$ values [e.g., the rain studies of Quartly et al. (1996, 1999)]. The AGC quantization was subsequently reduced to $0.0625 \mathrm{~dB}$. Further details of the intricacies of the TOPEX onboard pro- cessing are contained in Zieger et al. (1991) and Marth et al. (1993).

Figure 5a shows the shape of the mean waveforms for TOPEX-K $\mathrm{K}_{\mathrm{u}}$. The waveform is displayed in terms of telemetered bins (but with the received bin numbers provided at the top); thus, the apparent droop at the end of the displayed waveform is due to the nonlinear relation between telemetered bin number and time (or path delay). Along with the expected thermal noise level and wrap- 


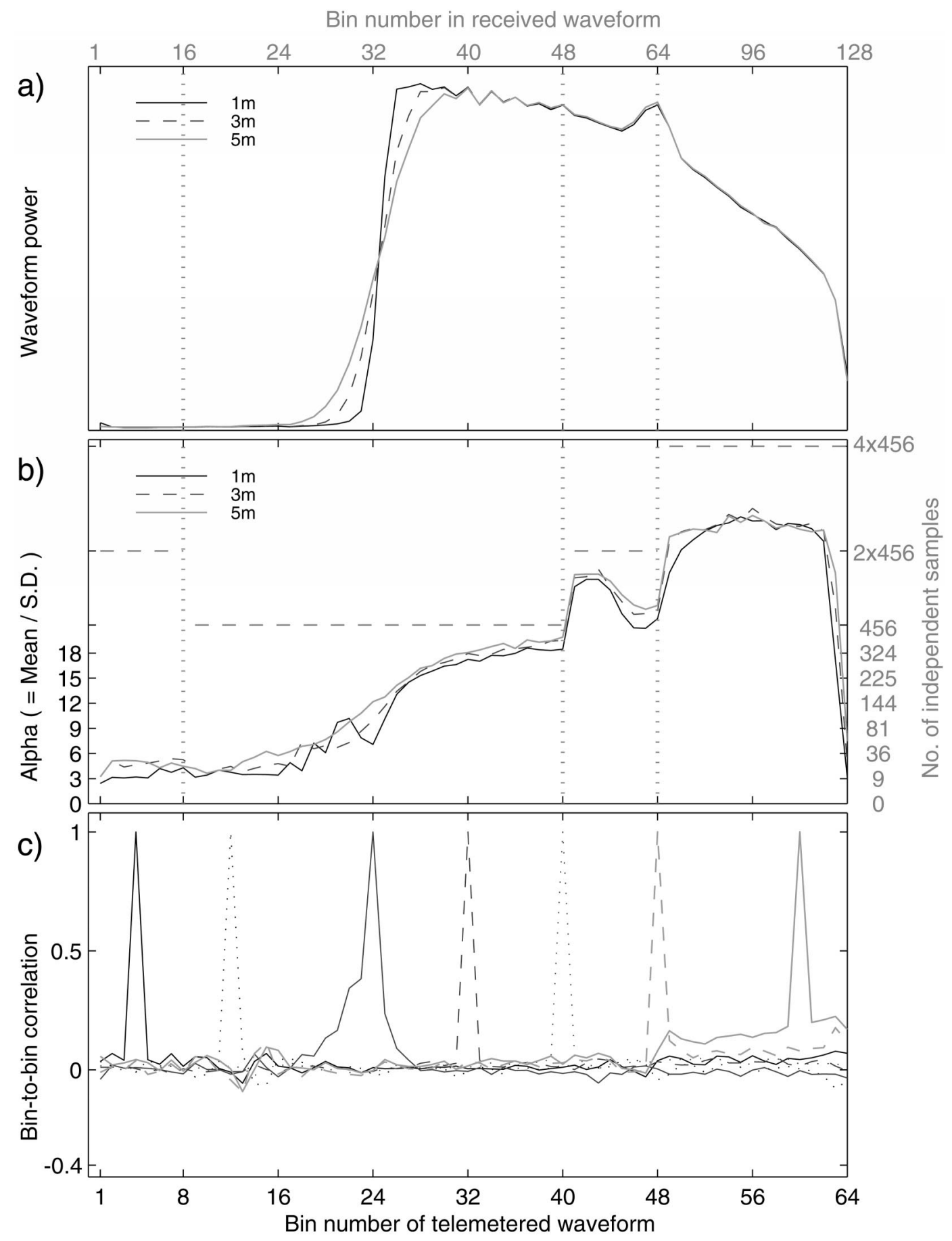

FIG. 5. TOPEX $\mathrm{K}_{\mathrm{u}}$ band. (Description same as for Fig. 3, except bin numbering of received waveforms also provided at top, and the vertical dotted lines show the changes in transcription from received to telemetered.)

around, these mean waveforms show a number of artifacts that relate to the instrument rather than the sea surface. A large "bump" occurs around received bin 65 (telemetered bin 49) due to a bias at the midpoint of the onboard DFT plus an "offset leakage" (Hayne et al. 1994). It is somewhat fortunate that the tracker was not designed to place the leading edge of the waveform at the halfway point of the window, as then there would have been a serious degradation in performance! A number of other lesser leakages are present in the waveform data; these have been detailed by Hayne et al. (1994) and Rodríguez and Martin (1994a). There is also a pronounced "sawtooth" variation 


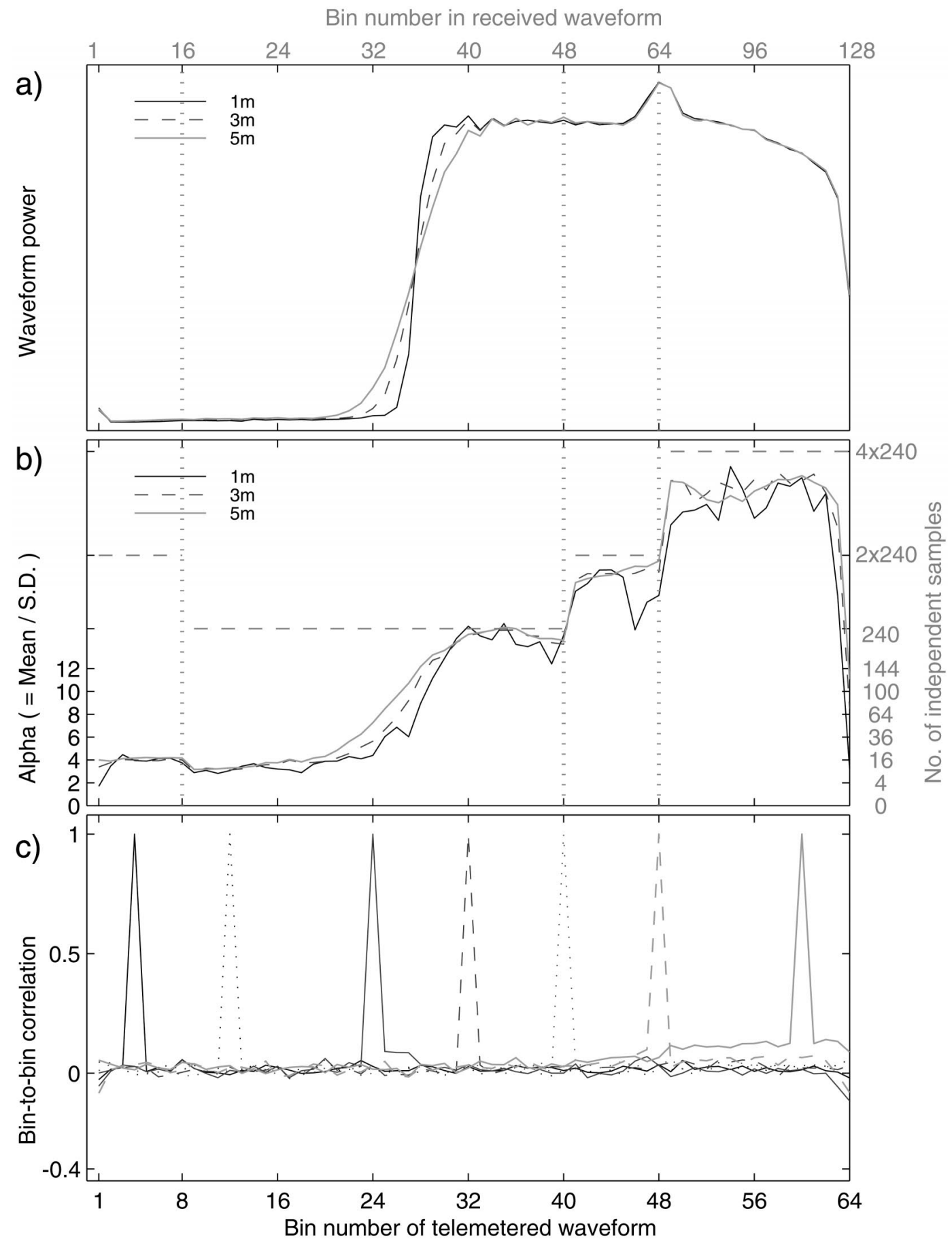

FIG. 6. TOPEX C band. (Description same as for Fig. 3, except bin numbering of received waveforms also provided at top, and the vertical dotted lines show the changes in transcription from received to telemetered.)

at the start of the trailing edge. Empirical adjustments to remove these effects have been detailed by Hayne et al. (1994); however, here we choose to show the data as they are actually received.

Hayne et al. (1994) and Rodríguez and Martin (1994a) have shown that the positions of these leakages vary according to whether the altimeter is progressing equatorward or poleward-essentially the implementation of the fine adjustment of the tracker depends upon whether the range rate is increasing or decreasing, for which the dominant factor is the oblateness of the earth. The leakage that occurs on the leading edge leads to a 
1-cm height bias between equatorward and poleward tracks (Rodríguez and Martin 1994a). Here we just give results for the equatorward passes.

The plots showing the number of independent pulses (Fig. 5b) are more complicated than those for ERS-1 (Fig. 3b). Principally, this is an effect of the averaging of successive bins in pairs or quartets, which, if anomalies in received bins are uncorrelated, will lead to an increase in $N$ by a factor of 2 or 4 . However, if that were the only effect, the $\alpha$ curves would be a series of plateaus similar to the dashed lines showing the maximum possible for the instrument. In the thermal noise region there are principally two plateaus: $\alpha=4.5$ for telemetered bins $1-8$, and $\alpha=3$ for bins 9-16. (This is actually clearer in the data for $\mathrm{C}$ band; see Fig. 6b.) It would be expected that the thermal noise in the electronic circuitry would be independent between received pulses; these low values for $\alpha$ are indicative of rounding caused by data quantization.

The number of independent samples increases as one moves through the leading edge and into the trailing edge. However, there is no step change into a plateau region, as there is for ERS-1 (Fig. 3b), but rather a continued increase. For early bins on the trailing edge, that part of the sea surface contributing to the return is a small disk or annulus, so that the various pathlengths from reflecting facets to altimeter do not decorrelate (change by the order of a wavelength) due to the satellite moving $\sim 1.3 \mathrm{~m}$ between pulses. For larger annuli (later bins) the pathlength decorrelates faster, but still not such that successive pulses are totally independent and the instrumental limit reached. Note that for bins around the tracker point the $\alpha$ curves for $H_{\mathrm{s}}=5 \mathrm{~m}$ are greater than for $H_{\mathrm{s}}=1 \mathrm{~m}$, as the larger wave height leads to contributions from a larger surface area (Chelton et al. 1989).

The sawtooth signature, evident at the beginning of the trailing edge in the mean waveforms, is much less pronounced, which is consistent with that effect being mainly multiplicative rather than additive error (see Table 1 of Hayne et al. 1994). The application of the bin averaging after telemetered bin 40 manifests itself as an increase in $\alpha$ of a little less than $\sqrt{2}$, which is consistent with those adjacent bins providing nearly independent information. The extra power in the mean waveform near bin 48 is principally an additive term uncorrelated with the actual true power in these bins. Consequently, the degree of independence drops markedly before a further increase due to greater bin averaging.

Thus, some of the information within TOPEX's many pulses is redundant at the wave heights considered here. Walsh (1982) provides a fuller explanation of the decorrelation between pulses due to satellite flight and suggests that the PRF adopted by TOPEX- $\mathrm{K}_{\mathrm{u}}$ is only fully exploited for wave heights of around $20 \mathrm{~m}$.

Figure 5c shows the bin-to-bin correlations between the various telemetered bins for TOPEX- $\mathrm{K}_{\mathrm{u}}$. There is minimal short-scale correlation, but a large-scale correlation in the later bins.

\section{2) TOPEX-C}

The design characteristics of TOPEX-C are obviously very similar to TOPEX-K $\mathrm{K}_{u}$, as they are both part of the same instrument. The principal differences are that every $53 \mathrm{~ms}$ TOPEX-C emits a burst of 60 pulses, with results from four bursts combined to give a mean waveform every $0.2 \mathrm{~s}$. The complicated averaging of 128 received bins to give 64 telemetered bins is the same as for TOPEX- $\mathrm{K}_{u}$; however, the nominal tracker point is 3 bins later than for TOPEX-K $\mathrm{K}_{\mathrm{u}}$.

The shape of the mean waveform for TOPEX-C (Fig. 6a) shows a much flatter trailing edge than TOPEX- $K_{u}$. This is because in using the same antenna the lower frequency has a much wider beamwidth (see Table 1) and consequently the flat surface response in (1) is much broader, leading to minimal tail-off within the short time segment recorded. Otherwise, features such as the offset leakage at received bin 65 and the sawtooth structure at the start of the trailing edge are very similar to TO$\mathrm{PEX}-\mathrm{K}_{\mathrm{u}}$.

The independence of the pulses given by the $\alpha$ curves of Fig. $6 \mathrm{~b}$ are similar to TOPEX-K $\mathrm{K}_{\mathrm{u}}$ (Fig. $5 \mathrm{~b}$ ) but simpler. The principal differences are that these curves reach their design limit (i.e., corresponding to the actual number of pulses emitted) for bins early in the trailing edge; and also that the effect of the offset leakage is much less pronounced. Each change in averaging of consecutive bins leads to a step change in $\alpha$ of slightly less than $\sqrt{2}$, probably due to a slight correlation in the values of consecutive bins.

Figure $6 \mathrm{c}$ again shows that there is negligible shortscale correlation, but apparently a slight large-scale correlation between the last 16 telemetered bins.

\section{3) Changes in TOPEX's Performance}

The TOPEX/Poseidon satellite has been operating for more than 8 yr now; however, during that time there have been significant changes in the characteristics of the TOPEX altimeter. Examination of the calibration data (G. S. Hayne and D. W. Hancock 1998, personal communication) showed that there had been a degradation in the point target response, in effect a broadening of the emitted pulse, with a raising of sidelobe levels. From cycle 150 onward, effects could be seen in the quality of the derived $H_{\mathrm{s}}$ and $\sigma^{0}$ values (G. S. Hayne and D. W. Hancock 1998, personal communication; P. Queffeulou 1998, personal communication; Quartly 2000). Consequently, on 11 February 1999 (cycle 236) operation was switched from side A of the TOPEX altimeter to side B (an identically constructed copy). Performance by side B was comparable to the early cycles of side A, confirming the belief that it was use rather than mere space exposure that had led to the deterioration of side A.

In addition to our use of early data from TOPEX side A, we also analyzed data from cycles 210 and 250 corresponding to before and after the changes in operation. 
For cycle 210, the mean TOPEX- $\mathrm{K}_{\mathrm{u}}$ waveform has the same basic shape (including the sawtooth structure and droop of trailing edge) as for early cycles; however, the power excess near telemetered bin 48 is much more pronounced, adding nearly $50 \%$ to the values in that region and clearly affecting any processing that tracks or normalizes by the peak values in a waveform. There is also significant power in the early bins from enhanced wraparound, and a slight raising in the thermal noise floor. Data from cycle 250 have a slightly smaller power spike near bin 48 than shown in Fig. 5a, but significant wraparound remains.

The power spike is characterized by increased variability, so the $\alpha$ curve shows a significant reduction there for cycle 210 . The increased mean signal in the thermal region (and consequent reduction in truncation effects) leads to increased values of $\alpha$ there for cycle 210 . Detailed examination of the bin-to-bin correlations reveals significant positive and negative correlations between telemetered bins 8-18 and 42-50. These correlations are absent in data from cycle 250, which again appears more free of artifacts than the data shown in Fig. 5c.

A very similar picture is found for TOPEX-C. Cycle 210 data are marked by a $50 \%$ power spike near bin 48 and significantly increased thermal noise, but no increase in the wraparound. There is no increased variability at the power spike, so the $\alpha$ curves are as before (Fig. 6b), except for significant raising in the thermal region. However, the whole of the waveform appears to be marked by significant correlations with many other parts. All these effects are removed by the change to side B operation.

\section{4) Poseidon}

The Poseidon altimeter emits $\sim 1700$ pulses per second and averages them in groups of 86 pulses every 53 $\mathrm{ms}$. The waveform is sampled in 64 bins of width 3.125 ns, with the tracker point at bin 31.5 , but the first two and last two bins (containing most of the wraparound effects) have been excised from the data stream. The AGC is applied in nominal steps of $1 \mathrm{~dB}$, with the same value applied for the whole of an 86-pulse group. In many cases the AGC value is found to oscillate rapidly between successive groups. As there are difficulties in combining waveform bins from different AGC settings (see appendix), we impose the additional flagging criterion that all waveforms used in a 10 -s segment must have the same AGC setting.

The shape of Poseidon's mean waveform (Fig. 7a) conforms closely to the theoretical model (Fig. 1a), without any striking artifacts. However, a slight waviness can be discerned along the trailing edge.

The $\alpha$ curves (Fig. 7b) are fairly constant for the trailing edge region, but show more variation with bin number than those for ERS-1 and ERS-2. Plateaus also exist in the thermal noise region, where the lower values are due to the quantization limiting the variability. Since the lower
$H_{\mathrm{s}}$ values are associated with the highest AGC settings, the power in the thermal noise region is lower in terms of integer counts (see Fig. 7a) and thus more affected by the data quantization. This is why the $\alpha$ curves show an $H_{\mathrm{s}}$ dependency in the thermal noise region. The dip in $\alpha$ at the beginning of the leading edge is due to increased variability in the mean waveforms in this region. This is consistent with the concept that the early returns from small disks will decorrelate slowly and thus lead to a lower number of independent pulses.

The plot of bin-to-bin correlations (Fig. 7c) shows the expected independence for bins in the trailing edge, but a surprising anticorrelation between adjacent bins in the thermal noise region. Although the effect of data quantization is severe in this region (for a wave height of $2 \mathrm{~m}$, the modal value is 2 counts, with a mean of $\sim 1.95$ ), this cannot in itself explain this observation, which is consistent across all of the thermal region.

\section{Summary and conclusions}

With the increasing demand for more accurate altimeters and the growth in applications of altimetry, there has been a need to reassess the quality of the data within the altimeter's own signal, rather than just examining atmospheric, tidal, and orbit corrections. Previous papers have examined waveforms from individual altimeters and shown them to differ from the theoretical model. Here we have examined five sources of altimeter waveform data from the last decade, showing the problems with each.

\section{a. Mean profile}

None have quite matched up to the expected shape for the mean waveform from an ocean surface. For the ERS altimeters no estimates of the thermal noise level are possible because of the data quantization. There is also a slight waviness along the trailing edge with a wavelength of about 8 bins. TOPEX's mean profile suffers from a number of artifacts-sawtooth signature at start of trailing edge, various power spikes, and also equatorward/poleward differences, all of which have been detailed by other authors (Hayne et al. 1994; Rodríguez and Martin 1994a). The magnitude of the power spike near telemetered bin 49 increased significantly with time, and, in combination with other anomalies, necessitated the switch to the redundant side of the TOPEX altimeter. The sawtooth signatures were also present in Seasat data [see Fig. 18 of Townsend (1980)].

The mean waveforms for Poseidon appear to be the closest to the expected shape, although they too exhibit a slight waviness along the trailing edge, but with a wavelength of around 16 bins. Bearing in mind that the emitted "pulses" are actually chirps, and that most of the onboard processing is performed in the frequency domain rather than the time domain (Chelton et al. 1989), it is likely that this waviness is due to "amplitude 


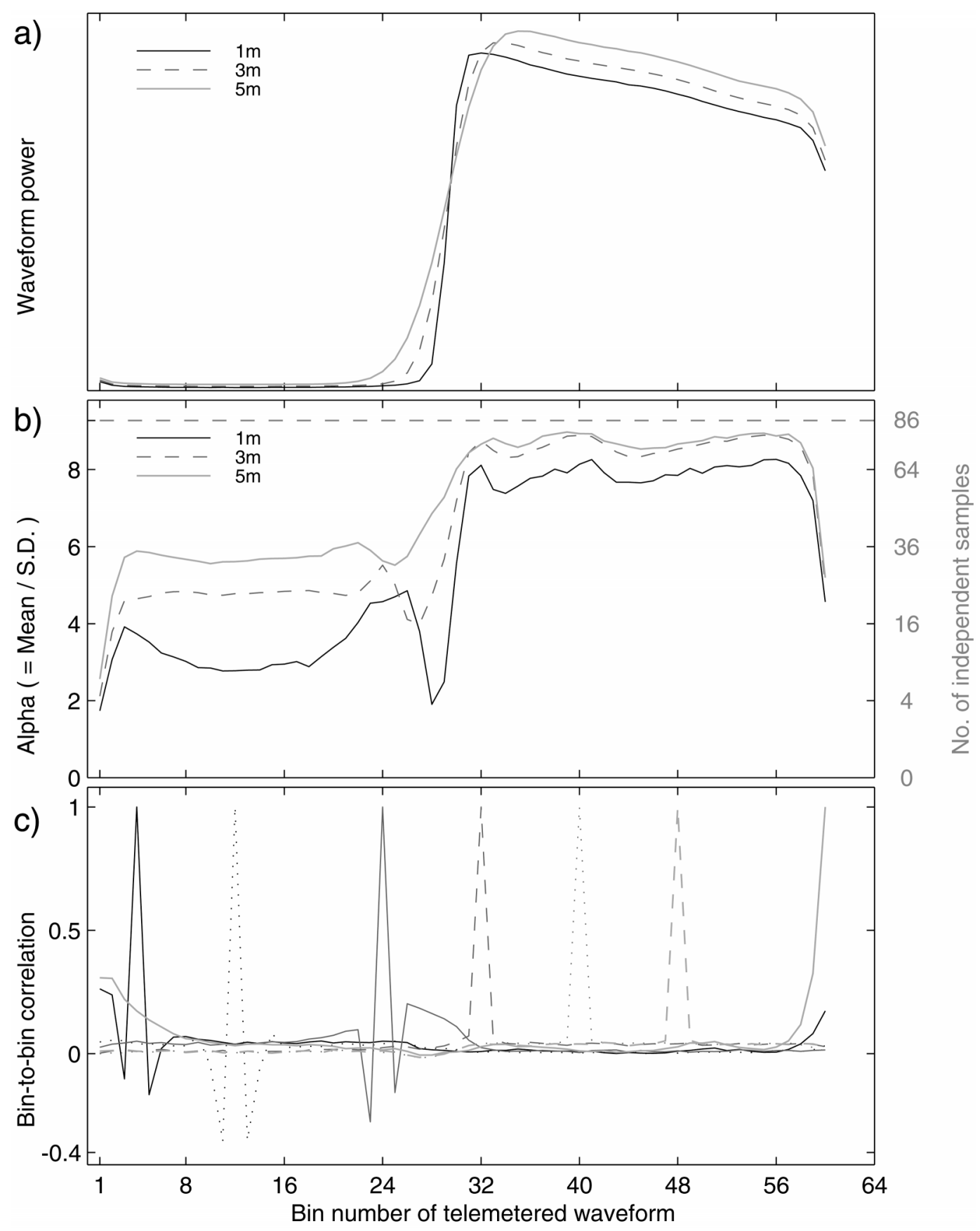

FIG. 7. Poseidon. (Description same as for Fig. 3.)

ripple," that is, a slight frequency-dependent gain in the amplifiers. A similar effect is seen in Geosat data (MacArthur et al. 1987). It would be preferable that the amplifiers have a very flat response rather than adjust bin values from calibration data, especially since for Seasat the gains in the calibration mode appeared to be different from those in tracker mode (Hayne and Hancock 1982). However, in the design of filters there has to be a compromise between the flatness in the pass region and the abruptness of the cutoff.
In some ways many of the artifacts in the mean profiles need not cause many problems in the geophysical retrievals, provided that the additive and multiplicative errors are known. However, for TOPEX there has been a general increase in the errors, and any correction can only be approximate. Thus, whether the waveforms are from over ocean, land, or ice, the errors in their interpretation are due to the uncertainties in the bin corrections rather than the corrections themselves. Such errors can make it hard to ascertain skewness of the ocean 
surface [as this has a small influence on the power at the start and end of the waveform's leading edge (Srokosz 1986)], or to determine the relative contribution of volume and surface scattering in ice studies. Particular problems exist for studies of rain, as the power in individual waveforms is subtracted from the local reference profile: if the rain has caused the tracker to drift, then sawtooth patterns could arise in the power anomalies. Broadening of the point target response (PTR) and power spikes within the leading edge both affect the determination of $H_{\mathrm{s}}$ over the ocean.

Much greater problems can occur if the existence of large power spikes affects the ability of the tracker to find and maintain lock on the surface. This is a particular problem when the received signal is non-Brown-like, as is the case for many land and ice applications. Alternatively, it could lead to more ocean data being flagged as contaminated by the presence of land within the footprint.

\section{b. Independence of pulses}

Berger (1972) showed how the effective number of independent pulses for an altimeter should be limited by the need for the satellite to have traveled far enough for the differential path delays to decorrelate. Experiments with variably spaced pulses from Skylab had suggested the decorrelation time to be $\sim 0.1 \mathrm{~ms}$ (Walsh 1982). However, for instruments in a higher orbit, and with a slower ground speed, the decorrelation time is longer. At moderate wave heights, Walsh (1982) suggests that the effective number of independent pulses per second at the tracker point will be 2000 for satellites at an altitude of $\sim 800 \mathrm{~km}$, and only 1500 at $1334 \mathrm{~km}$. The independence of pulses affects the height estimation (Berger 1972); however, it is not simply the value at the tracker point that matters, so much as the weighted average of the combination of bins used in the height determination (Rodríguez and Martin 1994b). For large values of $H_{\mathrm{s}}$, Rodríguez and Martin (1994b) deduced that a PRF of $4000 \mathrm{~Hz}$ would yield significantly lower height errors than a PRF of $2000 \mathrm{~Hz}$. For TOPEX-K our results show the number of independent pulses per second to be about 1500 at the tracker point, rising to about 3300 by bin 40 . The results in the later stages of the waveforms are harder to interpret, because of the averaging of successive bins and the major power spike near telemetered bin 49; however, they do indicate that the number of independent pulses is less near the end of the trailing edge than part way along it.

The number of independent pulses affects all geophysical retrievals, not just the determination of the tracker point. The maximum likelihood estimation method proposed for the ocean processing of ERS data (Tokmakian et al. 1994) was unusable, because it required the Rayleigh (fading) noise to be governed by the same statistics throughout: the truncation in the averaging process (see section $3 \mathrm{a}$ ) destroyed that assump- tion. The errors in rain retrievals are very closely related to the number of independent pulses (Quartly 1998).

Previous researchers had indicated that all the pulses were independent for Seasat (Lipa and Barrick 1981) and Geosat (Rodríguez and Martin 1994b), both of which only operated with a PRF of $1000 \mathrm{~Hz}$. In our analysis both Poseidon and ERS altimeters fall short of their design limit. This is unlikely to be a result of secular changes within the 10-s segments, as similar results were obtained for ERS-1 using 3-s segments, and our results for TOPEX-C agree with instrumental design. It is more likely to be due to the data quantization of the ERS and Poseidon altimeters. We know of no explanation for the structure observed along the trailing edge of Poseidon's $\alpha$ curves. Berger (1972) proposed the use of multiple receive antennas as a means of reducing fading noise without increasing PRF. However, extra antennas are not easily incorporated on the small altimetric satellites currently being planned. The $\alpha$ curves for TOPEX-C show that all 1200 pulses per second have independent errors at the start of the trailing edge, but again (when allowance is made for the averaging of consecutive bins) there is a slight droop toward the end of the trailing edge. These empirical observations of the level of fading noise as a function of bin number should guide the choice of waveform-fitting algorithms toward those that give greatest weight where $\alpha$ is largest.

\section{c. Bin-to-bin correlations}

For most altimeters the bin separation has been chosen to be close to the effective width of the emitted pulse, and so it is to be expected that the anomalies in the power received at adjacent bins should be uncorrelated, as they will be from different reflecting facets on the surface. (Observations close to the leading edge are ignored here, as it is difficult to remove the effect of waveform change due to variations in $H_{\mathrm{s}}$ or positioning by the tracker.) The ERS altimeters are an exception in that the applied smoothing after reception of the signal causes the effective pulse width to exceed the bin separation. This point is probably not fully appreciated by users of the altimeter data, as the documentation describing it has not been disseminated widely. Rodríguez and Chapman (1989) note that significant bin-to-bin correlations may also occur if the true PTR has much larger sidelobes than the idealized form. Our results for late in the side A phase of TOPEX are consistent with the observed change in the PTR (G. S. Hayne and D. W. Hancock 1998, personal communication).

Poseidon has a marked anticorrelation between neighbors in the thermal region, which we cannot explain. TOPEX- $\mathrm{K}_{\mathrm{u}}$ and TOPEX-C both show a broad low-level correlation between the anomalies in the distant bins (received bin 65 onward, i.e., those later in time than on the ERS or Poseidon waveforms). Although the effect of mispointing on the waveform is most prominent in the tail region of the waveform, it is a parameter that varies slowly (on the timescale of an orbit) and so cannot 
explain correlated changes within a 10 -s segment. Because these later bins represent reflections from annuli with radii around $10 \mathrm{~km}$, variations in the wind field of the direct track could be a possible explanation.

\section{d. Future altimeters}

Ideally, greater accuracy and usage of altimeter data will come from future altimeters being constructed to perform closer to the theoretical model, rather than considerable effort being expended to tune waveform coefficients for each individual altimeter. The next two altimetric satellites scheduled for launch are Jason-1 (the successor to TOPEX/Poseidon) and Envisat (successor to ERS-1 and ERS-2).

The altimeter on Jason-1 is called Poseidon-2, as it draws heavily on the experiences of Poseidon, but does include both $\mathrm{K}_{\mathrm{u}}$ - and $\mathrm{C}$-band frequencies, like TOPEX. It will sample the $\mathrm{K}_{\mathrm{u}}$ - and $\mathrm{C}$-band waveforms in 128 bins but only transmit $64 \mathrm{~K}_{\mathrm{u}}$-band and $60 \mathrm{C}$ band values to the ground, with the transmitted values corresponding to individual bins near the leading edge and to averages of groups of five elsewhere (P. Vincent 2000, personal communication). It is to be hoped that the use of solid-state amplification will lead to smooth mean waveforms, as for Poseidon. However, the principal operation of the instrument will be with 1800 $\mathrm{K}_{\mathrm{u}}$-band and $300 \mathrm{C}$-band pulses per second, so the PRF rather than the path decorrelation time will be a limit on the accuracy with which geophysical variables may be determined. (The rationale for the low $\mathrm{PRF}$ at $\mathrm{C}$ band is that that frequency is primarily used for the ionospheric correction, and its effect on the overall height accuracy is only one-sixth of that of the $\mathrm{K}_{\mathrm{u}}$-band signal.) Its use in other applications, where individual average waveforms are considered, will be compromised by the high level of Rayleigh noise produced by averaging only 15 pulses.

The altimeter on Envisat will be a modified form of that on its predecessors, having traveling wave tube amplification at $\mathrm{K}_{\mathrm{u}}$ band, but solid state used for its additional frequency at $\mathrm{S}$ band. ( $\mathrm{C}$ band could not be used because of conflict with other instruments on the Envisat platform.) The return pulses at both frequencies will be sampled in 128 bins, but the S-band information will be degraded by summing pairwise along the whole waveform to leave only 64 bins for telemetry. The instrument will emit $1800 \mathrm{~K}_{\mathrm{u}}$-band and $450 \mathrm{~S}$-band pulses per second, so again the performance will be limited by the PRF. The Envisat altimeter will also have the capability of recording and downloading a limited number of individual return pulses. The instrument is designed with flexibility in the location of the tracker point (the default being bin 32.5 of the 128 received bins); this should enable adjustment of the mean waveform position if the waveform suffers from power spikes as observed with TOPEX. Envisat will also have a different onboard tracker, designed to maintain lock across in- homogeneous terrain with rapidly changing height. This may, however, lead to more small movements of the tracker over normal ocean surfaces. In such cases waveforms would need to be retracked (repositioned within the window) prior to calculation of mean waveform shape. This could be a problem if the amplification is at all bin-dependent.

Despite these problems with existing and planned altimeters, measurements of sea surface topography, waves, and winds have been remarkably successful, and new applications (e.g., rainfall retrieval and land altimetry) have also emerged. However, altimetry applications are being pushed to the limit of existing systems, and improved measurement capability is only likely if some of the problems noted here are circumvented.

Acknowledgments. The ERS-1 and ERS-2 Waveform Product (WAP) was provided by ESA through NRSCL, the TOPEX Sensor Data Records (SDR) by Robert Benada and Liz Johnson of PO.DAAC at JPL, and the Poseidon waveform data by AVISO courtesy of Patrick Vincent. The Mullard Space Science Laboratory provided us with routines for handling the ERS and TOPEX data, and Steve Baker helped clarify many of the details of the ERS altimeters. Paolo Cipollini helped us understand the signal processing. An anonymous reviewer made us aware of several ESA technical reports, and Jerome Benveniste enabled us to obtain copies of them. We thank all these, without whose assistance this work would never even have been begun!

\section{APPENDIX}

\section{AGC Increments for Poseidon}

Each altimeter has an automatic gain control (AGC) that controls the amplification of the recorded signal, so as to best utilize the dynamic range of the detector and minimize the losses due to quantization. To achieve this, Poseidon uses a chain of attenuators, each of nominal value $1 \mathrm{~dB}$, with the attenuation fixed for each $0.05 \mathrm{~s}$ (86 pulses), so that each supplied average waveform has a single AGC value associated with it. This feature allows us to assess the incremental change between AGC settings.

As the AGC attempts to maintain the waveform signal within a fixed quantization range, one cannot simply compare global mean waveforms for an AGC setting of, say $19 \mathrm{~dB}$ to that of $20 \mathrm{~dB}$, because the geophysical signal (i.e., the roughness denoted by $\sigma^{0}$ ) of the two distributions will be different. However, there are sections where the AGC is oscillating between two values (see Fig. A1a). To automate the selection one can consider all transitions of the AGC from 19 to $20 \mathrm{~dB}$, and separately form the means of all the waveforms prior to and succeeding the transitions (Fig. A1b). For bins in the trailing edge, the ratio between the "before" and "after" waveforms is 1.244 , that is, an effective incre- 

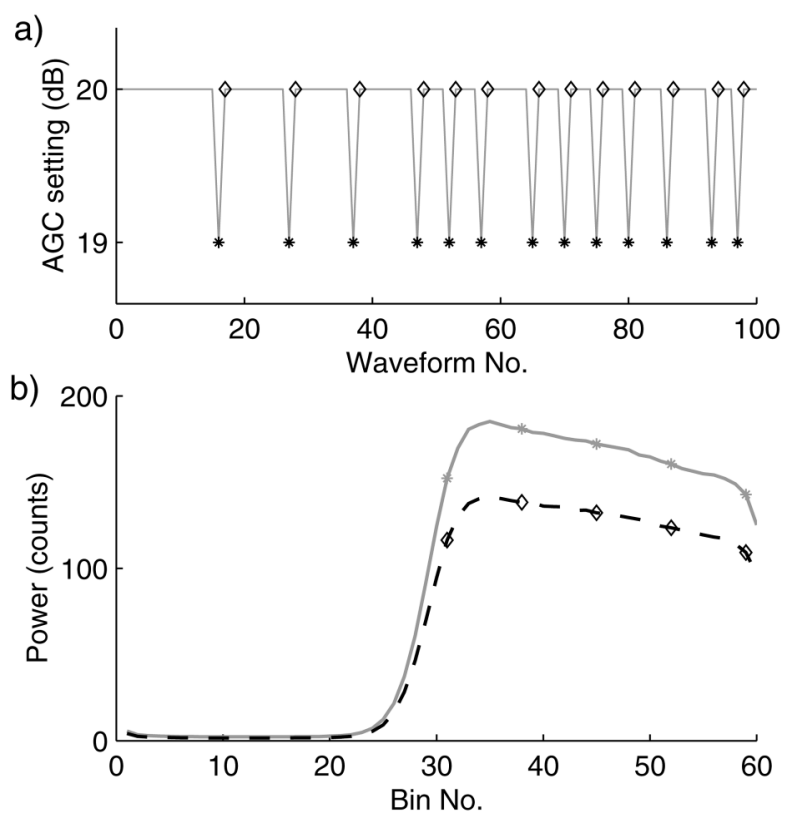

FIG. A1. (a) A 5-s series of AGC values for Poseidon. Instances prior to and succeeding a $19-$ to $20-\mathrm{dB}$ transition are marked by asterisks and diamonds, respectively. (b) Means of selected data from a whole day in cycle 186.

ment of $0.95 \mathrm{~dB}$. An almost identical value is derived from AGC transitions in the reverse direction, that is, from 20 to $19 \mathrm{~dB}$. Results for other transitions are given in Table A1.

The signal level in the thermal noise region $N_{\mathrm{T}}$ is the sum of detector noise $N_{\mathrm{D}}$, which should be independent of AGC setting, and receiver noise $N_{\mathrm{R}}$ (ambient microwave signals), which will be scaled by the AGC:

$$
N_{\mathrm{T}}=N_{\mathrm{D}}+N_{\mathrm{R}}\left(10^{-\mathrm{AGC}^{\prime} / 10}\right) \text {, }
$$

where $\mathrm{AGC}^{\prime}$ is an adjusted set of AGC values consistent with the increments in Table A1. Despite the severe quantization in the original waveforms, a plot of $N_{\mathrm{T}}$ against an exponential scale of AGC is fitted well by a straight line (see Fig. A2). The intercept gives $N_{\mathrm{D}}$ to be 0.6 counts. Data from a number of Poseidon cycles were analyzed and no drift in the relative scaling was detected.

TABLE A1. Observed changes in waveform power for each change in AGC setting. (See text regarding AGC values.)

\begin{tabular}{cc}
\hline \hline AGC change & $\begin{array}{c}\text { Actual increment } \\
(\mathrm{dB})\end{array}$ \\
\hline $16 \rightarrow 17$ & 0.80 \\
$17 \rightarrow 18$ & 0.99 \\
$18 \rightarrow 19$ & 1.15 \\
$19 \rightarrow 20$ & 0.95 \\
$20 \rightarrow 21$ & 0.87 \\
$21 \rightarrow 22$ & 1.24 \\
$22 \rightarrow 23$ & 1.29 \\
$23 \rightarrow 24$ & 0.89 \\
\hline
\end{tabular}

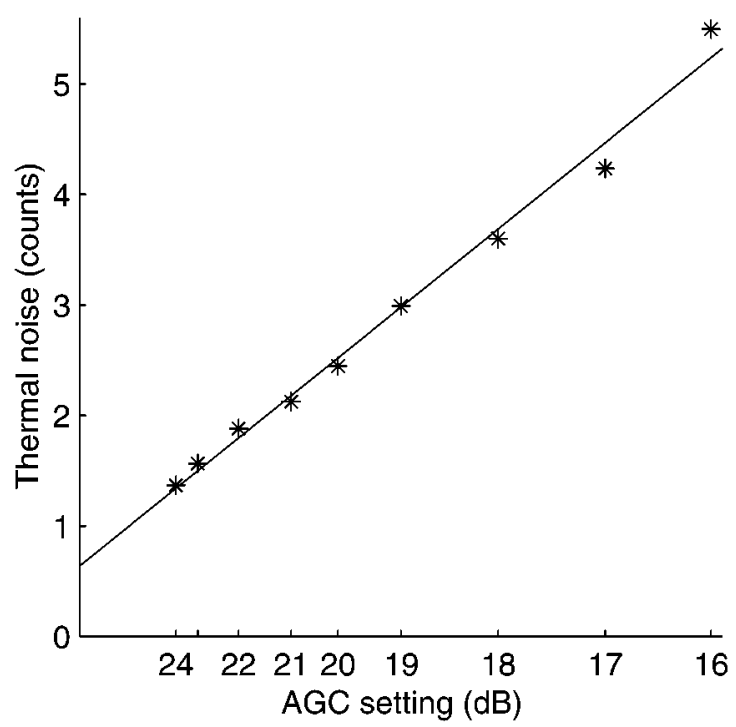

FIG. A2. Plot of power in thermal noise region (here average of bins 5-8) against adjusted AGC values on a logarithmic scale. (Data from cycle 186.)

\section{REFERENCES}

Bamber, J. L., 1994: Ice sheet altimeter processing scheme. Intl. J. Remote Sens., 15, 925-938.

Berger, T., 1972: Satellite altimetry using ocean backscatter. IEEE Trans. Antennas Propag., 20, 295-309.

Berry, P. A. M., 1999: Global digital elevation models-Fact or fiction? Astron. Geophys., 40, 310-313.

Birkett, C. M., 1994: Radar altimetry: A new concept in monitoring lake level changes. Eos, Trans. Amer. Geophys. Union, 75, 273275.

Brenner, A. C., R. A. Bindschadler, R. H. Thomas, and H. J. Zwally, 1983: Slope-induced errors in radar altimetry over continental ice sheets. J. Geophys. Res., 88, 1617-1623.

Brooks, R. L., D. W. Lockwood, and D. W. Hancock, 1990: Effect of islands in the Geosat footprint. J. Geophys. Res., 95, 28492855.

Brown, G. S., 1977: The average impulse response of a rough surface and its applications. IEEE J. Oceanic Eng., 2, 67-74.

Chelton, D. B., E. J. Walsh, and J. L. MacArthur, 1989: Pulse compression and sea level tracking in satellite altimetry. J. Atmos. Oceanic Technol., 6, 407-438.

Cudlip, W., J. K. Ridley, F. Strawbridge, A. Harris, and C. G. Rapley, 1994: Detecting surface roughness and moisture variations in deserts. Proc. Second ERS-1 Symp.-Space at the Service of Our Environment, Hamburg, Germany, European Space Agency, ESA SP-361, 849-853.

ESA, 1993: ERS-1 satellite to ground segment interface specification. ESA Document ER-IS-ESA-GS-0001, 588 pp. [Available from ESA-ESTEC, 2200 AG Noordwijk, Netherlands.]

Francis, R., 1990: RA characterisation data. ESA Document ER-TNESA-RA-0008, 10 pp. [Available from ESA-ESTEC, 2200 Noordwijk, Netherlands.]

_- and Coauthors, 1991: The ERS-1 spacecraft and its payload. ESA Bull., 65, 26-48.

Fu, L.-L., E. J. Christensen, C. A. Yamarone Jr., M. Lefebvre, Y. Ménard, M. Dorrer, and P. Escudier, 1994: TOPEX/POSEIDON mission overview. J. Geophys. Res., 99, 24 369-24 381.

Guymer, T. H., G. D. Quartly, and M. A. Srokosz, 1995: The effects of rain on ERS-1 radar altimeter data. J. Atmos. Oceanic Technol., 12, 1229-1247.

Guzkowska, M. A. J., C. G. Rapley, J. K. Ridley, W. Cudlip, C. M. 
Birkett, and R. F. Scott, 1990: Developments in inland water and land altimetry. ESA Contract Rep. 7839/88/F/FL, 422 pp. [Available from ESA-ESTEC, 2200 AG Noordwijk, Netherlands.]

Hayne, G. S., 1980: Radar altimeter mean return waveforms from near-normal-incidence ocean surface scattering. IEEE Trans. Antennas Propag., 28, 687-692.

— the SEASAT radar altimeter. J. Geophys. Res., 87, 3227-3231.

, C. L. Purdy, and P. S. Callahan, 1994: The corrections for significant wave height and attitude effects in the TOPEX radar altimeter. J. Geophys. Res., 99, 24 941-24 955.

Laxon, S. W., and C. G. Rapley, 1987: Radar altimeter data quality flagging. Adv. Space Res., 7, 315-318.

Legrésy, B., and F. Rémy, 1998: Using the temporal variability of satellite radar altimetric observations to map surface properties of the Antarctic ice sheet. J. Glaciol., 44, 197-206.

— _ — , and P. Schaeffer, 1999: Different ERS altimeter measurements between ascending and descending tracks caused by wind induced features over ice sheets. Geophys. Res. Lett., 26, 2231-2234.

Lipa, B. J., and D. E. Barrick, 1981: Ocean surface height-slope probability density function from SEASAT altimeter echo. $J$. Geophys. Res., 86, 10 921-10 930.

MacArthur, J. L., P. C. Marth, and J. G. Wall, 1987: The Geosat radar altimeter. John Hopkins APL Tech. Dig., 8, 176-181.

Marth, P. C., and Coauthors, 1993: Prelaunch performance of the NASA altimeter for the TOPEX/POSEIDON project. IEEE Trans. Geosci. Remote Sens., 31, 315-331.

Quartly, G. D., 1997: Achieving accurate altimetry across storms: Improved wind and wave estimates from C band. J. Atmos. Oceanic Technol., 14, 705-715.

_ 1998: Determination of oceanic rain rate and rain cell structure from altimeter waveform data. Part I: Theory. J. Atmos. Oceanic Technol., 15, 1362-1379.

_ 2000: Monitoring and cross-calibration of altimeter $\sigma^{0}$ through dual-frequency backscatter measurements. J. Atmos. Oceanic Technol., 17, 1252-1258.

— T. H. Guymer, and M. A. Srokosz, 1996: The effects of rain on Topex radar altimeter data. J. Atmos. Oceanic Technol., 13, 1209-1229.

— - M. A. Srokosz, and T. H. Guymer, 1999: Global precipitation statistics from dual-frequency TOPEX altimetry. J. Geophys. Res., 104, 31 489-31 516.
Raizonville, P., B. Cugny, O. Z. Zanife, Y. Jaulhac, and J. Richard, 1991: Poseidon radar altimeter flight model design and tests results. Proc. Intl. Symp. on Radars and Lidars in Earth and Planetary Sciences, Cannes, France, European Space, Agency, ESA SP-328, 93-98.

Ridley, J. K., and K. C. Partington, 1988: A model of satellite radar altimeter return from ice sheets. Intl. J. Remote Sens., 9, 601624.

Rodríguez, E., 1988: Altimetry for non-Gaussian oceans: Height biases and estimates of parameters. J. Geophys. Res., 93, 14 10714120.

_- and B. Chapman, 1989: Extracting ocean surface information from altimeter returns: The deconvolution method. J. Geophys. Res., 94, 9761-9778.

__ and J. M. Martin, 1994a: Assessment of the TOPEX altimeter performance using waveform retracking. J. Geophys. Res., 99, 24 957-24 969.

_ returns. IEEE Trans. Geosci. Remote Sens., 32, 553-561.

Srokosz, M. A., 1986: On the joint distribution of surface elevation and slopes for a nonlinear random sea, with an application to radar altimetry. J. Geophys. Res., 91, 995-1006.

Tokmakian, R. T., P. G. Challenor, T. H. Guymer, and M. A. Srokosz, 1994: The U. K. EODC ERS-1 altimeter ocean processing scheme. Intl. J. Remote Sens., 15, 939-962.

Tournadre, J., 1998: Determination of rain cell characteristics from the analysis of TOPEX altimeter echo waveforms. J. Atmos. Oceanic Technol., 15, 387-406.

Townsend, W. F., 1980, An initial assessment of the performance achieved by the Seasat-1 radar altimeter. IEEE J. Oceanic Eng., 5, 80-92.

Walsh, E. J., 1982: Pulse-to-pulse correlation in satellite radar altimeters. Radio Sci., 17, 786-800.

—_ F. M. Monaldo, and J. Goldhirsh, 1984: Rain and cloud effects on a satellite dual-frequency radar altimeter system operating at 13.5 and 35 GHz. IEEE Trans. Geosci. Remote Sens., 22, 615622.

Wingham, D. J., A. J. Ridout, R. Scharroo, R. J. Arthern, and C. K. Shum, 1998: Antarctic elevation change from 1992 to 1996. Science, 282, 456-458.

Zieger, A. R., D. W. Hancock, G. S. Hayne, and C. L. Purdy, 1991: NASA radar altimeter for the TOPEX/POSEIDON project. Proc. IEEE, 79, 810-826. 